\title{
Mehrsprachigkeit performativ in Szene gesetzt: ein diversitätsorientiertes Rap-Projekt
}

\author{
Stefan Blutner
}

\begin{abstract}
Zusammenfassung
Der Artikel beschäftigt sich mit der Frage, wie man Sprachenunterricht so gestalten kann, dass die sprachliche Diversität von Schüler*innen wertgeschätzt und aktiv einbezogen wird. Nach einigen einführenden theoretischen Überlegungen zu Mehrsprachigkeit, Macht und Schule wird das didaktische Konzept der identitätsbezogenen Sprachenbewusstheit erläutert. Dabei handelt es sich um eine sprachenübergreifende und sprachenverbindende Kompetenz, die es Schüler*innen ermöglichen soll, sich ihrer eigenen Sprachpraktiken und Einstellungen bewusst zu werden, über die Rolle von Sprachen in der Gesellschaft zu reflektieren und ihr sprachliches Handeln kritisch und kreativ weiterzuentwickeln. Am Beispiel eines Rap-Genres namens Conscious Rap wird daraufhin verdeutlicht, wie Künstler*innen ihre sprachliche Heterogenität ausdrücken und gegen gesellschaftliche Umstände behaupten. Im Mittelpunkt steht abschließend ein Unterrichtsprojekt, in dem Schüler*innen den Weg von der Betrachtung ihrer sprachlichen Umwelt über die Analyse von Rapsongs hin zur eigenen Kreation einer mehrsprachigen Performance einschlagen.
\end{abstract}

\section{Einleitung}

Lernende befinden sich im 21. Jahrhundert in mehrsprachigen, komplexen und dynamischen Räumen. Ihre Identitäten bilden sich und drücken sich auf ebenso komplexe Weise in der sozialen Interaktion mit ihren Familien, Mitschüler*innen, Lehrenden, Gemeinschaften, online, medial und von Angesicht zu Angesicht aus. Gerade in superdiversen Großstädten wie London und Berlin verwenden sie dabei ein sprachliches Repertoire, das entsprechend aktueller empirischer Forschung nicht mehr dem Konstrukt von standardisierten Nationalsprachen zuzuordnen ist.

Mit einem Rap-Projekt soll es Schülerinnen und Schülern ermöglicht werden, kreativ ihre individuelle Mehrsprachigkeit auszudrücken. Ihre Kultur- und Sprachhintergründe sind dafür essentieller Kern des Projekts. Es wird ihnen dabei erlaubt, im Sinne der Idee des Translanguaging Herkunftssprachen, Amtssprache des Landes und in der Schule erlernte Sprachen sowie deren verschiedenste 
Dialekte, Stile und Hybridisierungen zu kombinieren. Damit entsteht ein Raum, in dem sie als mehrsprachige Individuen ihre Identität performen. Diese kreative Aufgabe kann zu einer enormen Stärkung insbesondere der affektiven und identitäts-politischen Dimension von Language Awareness führen. Die Lernenden beschäftigen sich bewusst mit ihrem Sprachgebrauch und dem ihrer Peers, wobei sie gleichzeitig erfahren, dass ihre Art zu sprechen und Sprachstile zu kombinieren nicht nur legitim, sondern kunstvoll und engagiert sein kann.

In diesem Artikel soll zunächst die theoretische Grundlage für ein solches Rap-Projekt gelegt werden. Abschnitt 2 beschäftigt sich damit: Was bedeutet Mehrsprachigkeit überhaupt und wie steht sie in Zusammenhang mit Identität und Performativität? In Abschnitt 3 wird mit identitätsbezogener Sprachenbewusstheit eine kritisch-kreative Kompetenz vorgestellt, die als Ziel im Sprachunterricht anvisiert werden kann. Abschnitt 4 stellt Conscious Rap als ein musikalisches Genre vor, das zu diesem Ziel fruchtbar eingesetzt werden kann. In Abschnitt 5 wird abschließend der Ablauf eines diversitätsorientierten Rap-Projektes skizziert, das identitätsbezogene Sprachenbewusstheit auf performativem Wege fördert.

\title{
2 Mehrsprachigkeit als Performance von Identität
}

\begin{abstract}
Also, wenn du mich wirklich verletzen willst, dann beleidige meine Sprache. Sprachliche Identität ist die Kehrseite der Medaille ethnischer Identität - ich bin meine Sprache. Erst wenn ich auf meine Sprache stolz sein kann, kann ich auf mich selbst stolz sein. Erst wenn ich texanisches Chicano, Spanisch, Tex-Mex und all die anderen Sprachen, die ich spreche, als legitim anerkennen kann, kann ich meine eigene Legitimität anerkennen. Erst wenn ich zweisprachig schreiben und den sprachlichen Code wechseln kann, ohne ständig übersetzen zu müssen; solange ich Englisch oder Spanisch sprechen muss, wenn ich lieber Spanglish sprechen würde, und solange ich mich auf den Englischsprechenden einstellen muss und er sich nicht auf mich, so lange wird meine Sprache illegitim sein (zitiert in $\mathrm{Hu}$ 2003: 11)
\end{abstract}

Was bedeutet Mehrsprachigkeit heutzutage? Dieser Frage soziolinguistisch nachzugehen, ist die Grundlage für in diesem Artikel vorgestellte Konzepte und Unterrichtsvorschläge, die Mehrsprachigkeit als echte, lebensweltliche, soziale und identitätsbezogene Bedingung anerkennen und als ebensolche Fähigkeit fördern.

\subsection{Begriffe und Modelle von Mehrsprachigkeit}

Es gibt verschiedene Modelle, die individuelle Mehrsprachigkeit abbilden. Einige davon beziehen sich auf den Erwerb von Mehrsprachigkeit und unterscheiden beispielsweise zwischen dem simultanen und dem sukzessiven je nach Zeitpunkt des Erwerbs der Sprachen. Diese Modelle unterscheiden zudem oft nach der jeweiligen Sprachfähigkeit (siehe z.B. Bausch 2003). Manche 
Begriffe wie Semilingualismus oder Halbsprachigkeit weisen dabei jedoch eine starke Defizitorientierung auf, die ausblendet, dass die Sprecher*innen in ihrer Lebenswelt funktional kommunizieren können.

Von Relevanz ist ebenso die Unterscheidung nach Ursachen von Mehrsprachigkeit. Wolff (2010: 180f) differenziert zwischen enforced, also (durch Migration) erzwungener Mehrsprachigkeit, elitist, elitärer, seit früher Kindheit durch bilinguale Erziehung angestrebter Mehrsprachigkeit, instructed, schulisch angebahnter Mehrsprachigkeit, und geographically and socially induced, also durch eine plurilinguale Umgebung verursachter Mehrsprachigkeit.

Nicht vergessen sollte man, dass auch die Sprachpraktiken von Schüler*innen mit einer einzigen Kommunikationssprache heterogen sind. Wandruszka (zitiert aus dem Jahr 1997 in Oomen-Welke / Dirim 2013: 8) nennt dies ,innere Mehrsprachigkeit“. Zu betrachten sind sämtliche Variationen diaphasischer (nach Kommunikationssituationen variierende Stilebenen und Register), diastratischer (von verschiedenen gesellschaftlichen Gruppen gebrauchte Soziolekte), diatopischer (an verschiedenen Orten des Sprachgebiets gesprochene Dialekte) und sogar individueller Art (so genannte Idiolekte). Diese Varietäten sind teilweise so unterschiedlich, dass Tracy (2014) die Einteilung in Ein-, Zwei- und Mehrsprachigkeit aus linguistischer Sicht anzweifelt. Was als eigenständige Sprache und was als Varietät gelte, sei nicht naturgegeben, sondern Resultat politischer Prozesse.

\subsection{Sprache, Macht und Schule}

Am Beispiel der Institution Schule soll der Zusammenhang von Macht und Sprache verdeutlicht werden. Forscher*innen wie García \& Wei (2014) und Gogolin (2010) zeigen, dass Unterricht deutlich vom soziokulturellen Kontext geprägt ist. Gewählte und zugelassene Unterrichtssprachen offenbaren, wie eine Gesellschaft sich im Verhältnis zu anderen Gesellschaften definiert, welche kulturell-sprachlichen Identitäten erwünscht sind und welche Einstellungen gegenüber anderen dominieren. Diese Ideologien fasst Gogolin in ihren Arbeiten unter der Bezeichnung als monolingualen Habitus mit Blick auf das deutsche Bildungssystem zusammen.

Auch wenn Lehrer*innen und Forscher*innen immer wieder daran rütteln, sind folgende Tendenzen doch institutionell stark verankert:

Aus diesen Voraussetzungen ist eine gewisse Doppelmoral im Bildungssystem erwachsen. Je nach Prestige der gesprochenen Sprachen, wird die Mehrsprachigkeit eines Kindes entweder als Kapital oder als Gefahr und Hindernis betrachtet (siehe Tracy 2014: 20). Die Fähigkeiten von mehrsprachigen Kindern, die keine mit Prestige verbundenen Sprachen sprechen oder in ihren Sprachen nicht der bildungssprachlichen Standardnorm entsprechen, werden nicht wertgeschätzt. Und ebenso haben diejenigen einsprachigen Kinder mit Vorurteilen zu kämpfen, wenn sie in dieser nicht in der Lage sind, schriftsprachliche Standards zu erreichen. 
Tabelle 1: Die Monolingualismus- und Standard-Ideologie der (deutschen) Schule

\begin{tabular}{l|l}
\hline $\begin{array}{l}\text { National- } \\
\text { sprachentum }\end{array}$ & $\begin{array}{l}\text { Als vollwertige Sprachen gelten nur Nationalsprachen, insbesondere jene, die einem Nationalstaat } \\
\text { eindeutig zuzuordnen sind (z.B. Deutsch-Deutschland). }\end{array}$ \\
\hline $\begin{array}{l}\text { Euro- } \\
\text { zentrismus }\end{array}$ & $\begin{array}{l}\text { Europäische Nationalsprachen genießen höheres Prestige als Nationalsprachen typischer } \\
\text { Einwanderungsländer in Afrika und Asien. Dabei sind west- und nordeuropäische } \\
\text { Nationalsprachen deutlich prestigeträchtiger als südosteuropäische. }\end{array}$ \\
\hline $\begin{array}{l}\text { Englische } \\
\text { Dominanz }\end{array}$ & $\begin{array}{l}\text { Die Vormachtstellung des Englischen wird als Sprache der wirtschaftlichen Globalisierung } \\
\text { verteidigt und ausgebaut. }\end{array}$ \\
\hline $\begin{array}{l}\text { Standard, } \\
\text { sonst nichts! }\end{array}$ & $\begin{array}{l}\text { Akzeptiert wird jeweils nur die nationale Standardnorm einer Sprache. Andere Varietäten werden } \\
\text { ignoriert oder als schädlich für den Spracherwerb missbilligt. Die bildungssprachliche } \\
\text { Beherrschung der Amtssprache des Landes wird oft schon bei jungen Schüler*innen } \\
\text { vorausgesetzt. }\end{array}$ \\
\hline $\begin{array}{l}\text { Puristische } \\
\text { Trennung }\end{array}$ & $\begin{array}{l}\text { Der Rückgriff auf Erstsprachen oder Sprachen, die nicht Zielsprache sind, wird vermieden. } \\
\text { Übersetzungen oder code-switching (das Hin- und Herwechseln zwischen Sprachen in einer } \\
\text { Äußerung) werden missbilligt oder gar sanktioniert. }\end{array}$ \\
\hline
\end{tabular}

\subsection{Sprache, Macht und Identität}

Welche Auswirkungen haben die geschilderten sprachpolitischen Voraussetzungen auf Lernende? Mehrsprachigkeit hat ganz materielle Folgen, denn Sprachen und Sprachvarietäten mit hohem Prestige ermöglichen Zugang zu Arbeit, Handel und Institutionen, die denjenigen verschlossen bleiben, die diese nicht beherrschen. Dominante Sprachpraktiken sind Voraussetzung für akademischen und ökonomischen Erfolg (siehe García \& Wei 2014: 48).

Aus Sicht von migrationsbedingt mehrsprachigen Schüler*innen bedeutet dies oft eine diskriminierende Realität. Ihre Mehrsprachigkeit ist nicht frei gewählt und sie eröffnet keine Perspektiven, sondern wird im Gegenteil bestraft. „Heutzutage ist die Bestrafung nicht körperlich, sondern funktioniert durch Unterricht und Bewertungen, die monolingualen Sprachstandards folgen und dafür sorgen, dass bilinguale Schüler schlechtere Noten bekommen, sich unangebracht fühlen und in den Schulen versagen" (García \& Wei 2014: 56) (vom Autor übersetzt).

\subsection{Sprache als Performance}

Das Zitat zu Beginn dieses Abschnitts soll verdeutlichen, dass wenn man bestimmte Teile der Identität von Lernenden durch gesellschaftlich dominante Diskurse und Prozesse hegemonisch delegitimiert, während andere legitimiert werden, es wahrscheinlich ist, dass diese „einen Sinn von Unterlegenheit internalisieren, der potentiell ihr Selbstwertgefühl und ihre akademischen Selbsterwartungen beschädigt" (Cummins, zitiert aus dem Jahr 2001 von Breidbach et al. 2011: 12). Ein Gefühl von Versagen und Mangel an Selbstbewusstsein sind die Folge.

Andererseits offenbart das Zitat jedoch auch, dass im Sprachgebrauch eines Individuums dessen Identität voll zum Ausdruck kommen kann. Für 
Schüler*innen sind hybride Sprachpraktiken oft stolzer Ausdruck dessen, dass sie „eine Menge Kultur haben“ und in Toleranz leben möchten (Low \& Sarkar 2014: 102) (vom Autor übersetzt). Sie kreieren, konstruieren und verhandeln ihre Identitäten auf der Basis sprachlicher Ressourcen, drücken damit Zugehörigkeit, Solidarität, Distanz u.v.m. aus (siehe Tracy 2014: 16). Sprachliche Ressourcen sind Teil ihres „identity repertoire“ (Pennycook 2003: 517), einer Fülle von sprachlichen und nicht-sprachlichen, also allgemein semiotischen, multimodalen Elementen, die sie in ihrem Handeln und Verhalten nutzen, um sich selbst zu definieren und präsentieren.

Gogolin (2004: 57) stellt fest, dass für Mehrsprachige Herkunftssprachen und Majoritätssprache meist nicht in konkurrierender Beziehung zueinanderstehen, sondern als einander komplementierende Kommunikationssysteme konzeptualisiert werden. Der Prozess der Identitätsfindung ist für viele dabei nicht einfach, da sie sich zwischen Kulturen und Sprachen verortet fühlen und neue transkulturelle und transsprachliche Identitäten finden müssen, die sich sowohl am Ort ihres Aufenthalts lokal als auch mit starken Verbindungen zur ihren Herkunftskulturen bilden (siehe Higgins 2001: 8f; Holmes 2015: 12). Fest steht: Durch Sprachen performen Menschen ihre eigene Identität.

\section{Identitätsbezogene Sprachenbewusstheit als Ziel von Sprachunterricht}

In diesem Abschnitt soll nun ein sprachendidaktisches Konzept vorgestellt werden, welches ich im Zuge meiner Masterarbeit (Blutner i.E.) entwickelt habe. Dieses Konzept soll es Sprachlehrer*innen erlauben, Sprachförderung mit Identitätsförderung zu verbinden. Es basiert auf einschlägigen Forschungserkenntnissen aus den Gebieten der Mehrsprachigkeits- und Sprachenbewusstheitsforschung der letzten Jahre.

Laut Ellis (2012: 17) entstand Sprachenbewusstheit im englischen Sprachraum als Language Awareness unter anderem unter der Feder von Eric Hawkins (1984), der es als mehrsprachiges Konzept anvisierte, das gezielt die Erstsprachen der Lernenden miteinbeziehen sollte. Hawkins Grundgedanke war, dass Sprachenbewusstheit „eine Brücke zwischen allen Aspekten von Spracherziehung (Erstsprachen, Fremdsprachen, Zweitsprachen, Minderheitensprachen, klassische Sprachen), die derzeit in Isolation stattfinden", herstellen sollte (Hawkins, zitiert in García 2008: 387) (vom Autor übersetzt). Die Association for Language Awareness definiert Sprachenbewusstheit heute als "explizites Wissen über Sprache und bewusste Wahrnehmung und Sensibilität beim Sprachenlernen, -unterrichten und -gebrauch“ (siehe Ellis 2012: 2) (vom Autor übersetzt).

Die untenstehende Grafik zeigt das von mir entwickelte Modell von Sprachenbewusstheit. Möchte man Sprachenbewusstheit fördern, so kann man zwischen verschiedenen Blickwinkeln, Dimensionen, Wissensebenen und sprachlichen Ebenen unterscheiden: 


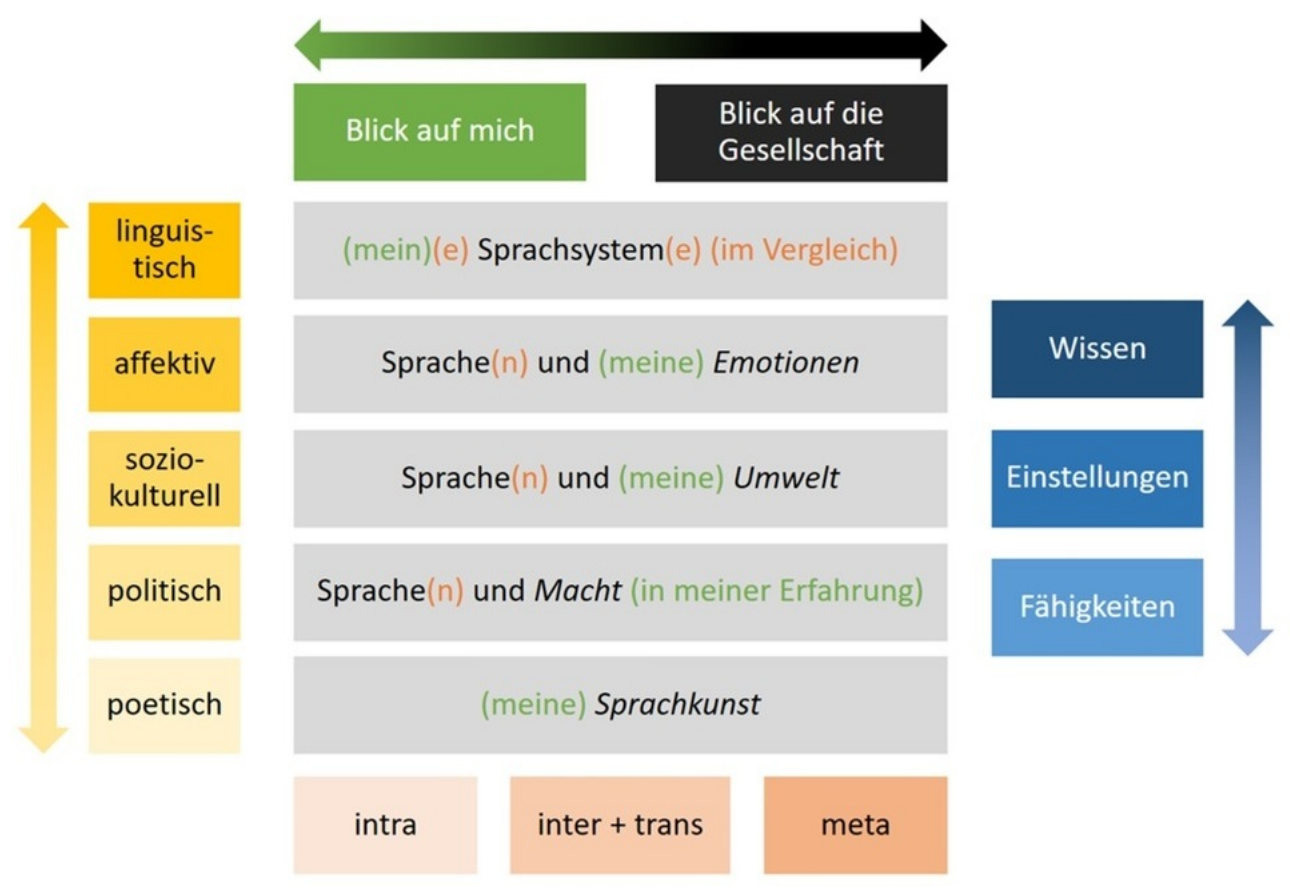

Abbildung 1: Gesamtmodell von Sprachenbewusstheit (Blutner i.E.)

Mehrsprachige Praktiken können aus zwei Perspektiven betrachtet werden: aus subjektiver Perspektive, also dem Blick nach innen, auf das Ich, oder mit dem Blick nach außen bzw. aus der Vogelperspektive auf die Gesellschaft. Interessiere ich mich für meine eigenen sprachlichen Praktiken oder für das Phänomen Mehrsprachigkeit in der Gesellschaft? Diese Perspektiven existieren natürlich nicht getrennt, sondern ergänzen sich gegenseitig. Wie im vorangegangenen Abschnitt beschrieben, ist insbesondere der subjektive Blick stark von gesellschaftlichen Verhältnissen (z.B. Sprachideologien, -hierarchien, und -vorurteilen) geprägt.

Sprachenbewusstheit lässt sich zudem auf drei Wissensebenen verorten: den Ebenen des deklarativen Wissens, der Einstellungen und der Fähigkeiten. So kann ich wissen, welche Sprachen in Deutschland gesprochen werden und wie viele Sprecher sie haben (Beispiel für deklaratives Wissen), kann Standardsprachlichkeit und Korrektheit als erstrebenswert betrachten (Beispiel einer Einstellung) und kann in der Lage sein, meinen sprachlichen Stil gemäß Situation und Gesprächspartner anzupassen (Beispiel einer Fähigkeit). Die drei Wissensebenen hängen zusammen: Fähigkeiten sollten auf Grundlage von Wissen reflektiert werden. Und andersherum sollte der Erwerb von Wissen über die eigene oder gesellschaftliche Situation in veränderte Einstellungen und folgerichtiges Handeln münden.

Fünf Dimensionen der Betrachtung von Sprache können unterschieden werden: (1) die sprach-strukturelle Dimension (d.h. wie sind Sprachen grammatikalisch aufgebaut, welchen internen Regeln folgen sie), (2) das 
Verhältnis von Sprache und Gefühlen (affektive Dimension), (3) das Verhältnis von Sprache und Umwelt (soziokulturelle Dimension) und (4) das Verhältnis von Sprache und Macht (politische Dimension). Das Bewusstsein dafür, wie mit Sprache gespielt und experimentiert werden kann, wie Sprache ästhetisch verwendet werden kann, um beispielsweise Rhythmus oder Reime zu erzeugen, und dies nicht nur innerhalb einer Sprache, sondern sogar zwischen den Sprachen, nenne ich poetische Dimension von Sprachenbewusstheit (5).

$\mathrm{Zu}$ guter Letzt gibt es verschiedene Sprachebenen, die betrachtet werden können: Interessieren mich nur Aspekte einer ausgewählten Einzelsprache, bewege ich mich auf der intralingualen Sprachebene. Möchte ich verschiedene Sprachen miteinander vergleichen, befinde ich mich auf der interlingualen Sprachebene. Weckt insbesondere die Vermischung von Sprachen in CodeSwitching- bzw. Translanguaging-Praktiken meine Neugierde, interessiert mich die translinguale Ebene. Und betrachte ich sprachenübergreifende Phänomene, die für alle Sprachen gelten, so heißt dies, ich arbeite auf metalingualer Ebene.

Die vielen Bereiche von Sprachenbewusstheit, die sich aus der Kombination dieser Wissensebenen, Sprachebenen, Dimensionen und Perspektiven ergeben, können in diesem Artikel nicht erschöpfend dargestellt werden. Das Projekt, das im abschließenden Abschnitt präsentiert wird, konzentriert sich auf eine interessante Schnittmenge dieser Bereiche, die ich als Identitätsbewusstheit bzw. Identity Awareness bezeichne (Blutner i.E.). Hierbei geht es darum, dass Schüler*innen ihre eigene Mehrsprachigkeit entdecken, erkunden und entwickeln. „Was bedeuten die sprachpolitischen Voraussetzungen in meinem Land für meine Identität?" ist beispielsweise eine Frage, die Intersektionen zwischen dem Blick nach außen und innen sowie zwischen affektiver und politischer Dimension von Sprachenbewusstheit eröffnet. Die poetische Dimension in Verbindung mit der translingualen Ebene von Sprachenbewusstheit erlaubt es Schülern, Normen vom Sprachgebrauch zu verlassen, andere Sprachpraktiken auszuprobieren und eigene, hybride und experimentelle Praktiken zu erschaffen, die ihre individuelle Identität ausdrücken.

Bei diesem Begriff von Sprachenbewusstheit handelt es sich demnach um eine kritisch-kreative Kompetenz. Zusammengefasst ist das Ziel von Sprachenbewusstheit ein emanzipatorisches Bildungsanliegen, nämlich die Fähigkeit, die eigene sprachliche Identität im Kontext von sprachlich-diskursiv geprägter Gesellschaft und Macht zu reflektieren, zu behaupten, auszuhandeln und kreativ zu entwickeln. Dies bedeutet, Fähigkeiten reflexiv einzusetzen, um das eigene Selbst- und Weltbild regelmäßig zu hinterfragen, aktiv zu konstruieren und in bewusste Handlungen als Subjekt in der Gesellschaft zu übertragen.

\section{Conscious Rap als performatives Ausdrucksmittel}

Was in den bisherigen Abschnitten erläutert wurde, mag auf den ersten Blick abstrakt wirken. Interessanterweise gibt es jedoch ein musikalisches Genre, in dem Künstler*innen die identitätsbezogene Komponente von Sprachenbewusstheit schon seit Jahrzehnten aufblühen lassen, der so genannte Conscious 
Rap. Mit diesem Medium kann es gelingen, Schüler*innen für die komplexen Verbindungen von Sprache, Identität und Macht zu sensibilisieren, und sie selbst zu kritisch-kreativem Handeln anzuregen.

Rap ist die musikalische Kunstform der HipHop-Kultur, der auch Graffiti, DJing und Breakdancing zugeordnet werden. HipHop entstand in der Bronx von New York in den 70er Jahren. Seine Wurzeln sind kulturell vielfältig und speisen sich aus der Kultur der dort lebenden Afroamerikaner*innen und Einwanderer*innen aus Puerto Rico und anderen Teilen der Karibik. Ein großer Einfluss wird der oralen, afrikanischen Kultur der Griots zugerechnet. Dalzell (2014: 21) fasst gut zusammen, welche Bedeutung diese orale Tradition für den Rap hat; Rappen bedeutet demnach die Erfüllung vielfältiger kommunikativer und über Kommunikation hinausgehender Funktionen:

Wie Rapper sind Griots mehr als Geschichtenerzähler. Als Wortkünstler handeln sie als Historiker, Genealogen, Diplomaten, Vermittler, Übersetzer, Lehrer, Ermahner, Zeugen, Überlieferer, Berater, Schlichter, Anpreiser, Schwätzer, Satiriker und politische Kommentatoren. (vom Autor übersetzt $)^{1}$

Aufgrund des Mangels an ökonomischen Ressourcen, ihrer sozial niedrigen Stellung und rassistischer Ausgrenzung war es den Menschen aus der Bronx verwehrt, Diskotheken zu besuchen oder an anderen kulturellen Praktiken teilzunehmen, sodass sie in diesen Vierteln selbst als Kulturschaffende aktiv wurden. Seit den 80er Jahren fand die HipHop-Kultur daraufhin globale Verbreitung. Überall wird heute HipHop lokalisiert und rekontextualisiert, d.h. mit lokalen Themen, Bräuchen, Sprachen, Musikinstrumenten und Stilen kombiniert.

Aus ästhetischer Perspektive definiert Lensch (2014: 49) Rap als „skandierende[n] Sprechgesang über einem Beat". Ein Beat bezeichnet dabei den gesamten instrumentalen Teil eines Musikstückes, der wahlweise mit Instrumenten oder am Computer elektronisch erzeugt werden kann. Diesem instrumentalen Teil zugrunde liegt meist ein aus 4 Perkussionselementen bestehender Rhythmus, der sich durch den Song hindurch wiederholt und den metrischen Puls des Musikstücks bildet. Auch diese kleineren musikalischen Einheiten werden als Beats bezeichnet. Aufgrund der globalen Verbreitung von Rap verschmelzen Beats mit lokalen Kulturen: Rhythmen, Melodien, Instrumente und musikalische Zitate aus verschiedensten Stilen wie Jazz, Reggea, Salsa, Flamenco u.v.m. finden Eingang in die Gesamtkomposition.

Typischerweise ist ein Rapsong in Strophen (Verses) (nicht zu verwechseln mit den Versen eines Rapsongs, die man Lines nennt) und Refrain (Chorus bzw. Hook) gegliedert und kann zusätzlich eine Bridge (eine musikalische Überleitung) enthalten. Nicht selten kommt es vor, dass die Verses rhythmisch gesprochen werden, während der Hook gesungen wird. Dafür arbeiten MCs (ein anderes Wort für Rapper*innen) und Sänger*innen gelegentlich zusammen.

\footnotetext{
${ }^{1}$ Aus Gründen der Lesbarkeit wurde in dieser Übersetzung auf die Hinzufügung des GenderSuffixes *innen verzichtet.
} 
Der Sprechgesang eines/einer Rapper*in muss mit dem Beat harmonieren. Dem Zeit- und Rhythmusempfinden ordnen sich Bedeutungen oft unter, beobachtet Lüdtke (2007: 97). Flow bezeichnet das rhythmische Sprechen im und über den Takt hinweg. Eine Person, die guten Flow hat, überzeugt mit ihrem Sinn für Rhythmus und Timing.

Struktur erzeugen am Ende (oder oft auch innerhalb) einer Line die Reime (Rhymes), das wichtigste Element eines Rapsongs. Für einen Reim braucht es nicht perfekte Übereinstimmungen von Silben, oftmals reicht schon eine leichte Homophonie oder gar die gleiche Anzahl an Silben oder das gleiche Intonationsmuster aus (siehe Alim 2009). Die Wichtigkeit von Metrik bildet eine Parallele zwischen Rap und Lyrik. Dies, die Einteilung in Verses (Strophen) und die Verwendung weiterer poetischer Stilmittel in der Sprache des Rap (wie z.B. Metaphern, Parallelismen, Chiasmen, Alliterationen, Sprachspiele etc.) hat dazu geführt, dass Rap auch unter dem Namen Street Poetry bekannt ist.

Conscious Rap ist ein Genre des Rap, das sich von anderen Genres wieParty oder Gangsta Rap abgrenzt. Es unterscheidet sich vor allem durch seine Themenwahl: Im Vordergrund stehen Gender, Ethnizität und sozialer Hintergrund, Migrationsund Diskriminierungserfahrungen, Spannungen in der Gesellschaft sowie die Suche nach neuen Räumen und Identitäten. Von sprachlicher Diskriminierung von Menschen oder Gruppen und Gewaltverherrlichung nimmt dieser Rap Abstand. Aus diesen Gründen eignet er sich hervorragend für die pädagogische Arbeit und kann Schüler*innen als Vorbild dienen.

Als Fallbeispiel soll hier der Song „Vit Ki”u“ von Fawng Daw dienen. Den Song kann man auf youtube.com (Daw 2012) hören und in Anhang A nachlesen. Er behandelt autobiographisch mehrere Migrationserfahrungen eines vietnamesischstämmigen Jugendlichen auf der Suche nach seiner Identität, der zunächst mit seiner Familie in Vietnam aufwuchs, danach nach Deutschland und schließlich in die USA übersiedelte. „Vit Ki ' $\mathrm{u}^{\text {“ bedeutet }}$ „Überseh-Vietnamese“.

Der Song hat eine sehr klare Struktur. Der sich wiederholende Hook ist gänzlich auf Englisch (phonetisch und lexikalisch deutlich von der HipHopNation-Language geprägt, einer im HipHop-Milieu benutzten Sprachvarietät) gehalten und besingt die Gespaltenheit der Identität als „child of two worlds“ (Kind zweier Welten), das im Osten geboren und im Westen aufgewachsen ist („Born in the east, raised in the west"). Die drei Verses verhandeln nacheinander zunächst die Identität als „resident" in den USA (Verse 1) mit Wurzeln in Vietnam (Verse 2), der auch seine Freunde und Jugend in Deutschland nicht vergessen hat (Verse 3). Interessant ist die interne Struktur der Verses. Während Verse 1 komplett auf Englisch erzählt wird, sind Verses 2 und 3 jeweils in der Mitte gespalten zwischen Vietnamesisch und Englisch bzw. Deutsch und Englisch. Der vietnamesisch- bzw. deutschsprachige Teil richtet sich dabei an ein spezifisches Publikum, nämlich die vietnamesischen Bekannten und Verwandten in Vietnam respektive die verbliebenden Freund*innen und Bekannte in Deutschland.

Alles in allem ist sein Rap eine Selbstdarstellung, in der Fawng Daw durch geschickte Sprachwahl allen Stationen seiner Biographie Tribut zollt und gerecht 
zu werden versucht. Er versprachlicht die Widersprüche und Schwierigkeiten, die ihm durch seine transnationale Identität entstanden sind, doch konstruiert daraus ein positives Selbstbewusstsein („I am who I am / There is nothing I need to change") als Botschafter (,ambassador") der vietnamesischen Diaspora. Seine letzte Line „So you can call me the ambassador" bringt den Prozess der Identitätsfindung auf den Punkt. Nach vielen externen Zuschreibungen, die für ihn diskriminierend waren oder zwischen denen er als Transnational keine Identität fand, gibt er sich im performativen Akt des Raps nun selbst den Namen des Botschafters „Vịt Ki` u“.

\section{Beschreibung des Rap-Projekts}

In Abschnitt 5 soll nun ein Projekt skizziert werden, das kritisch-kreatives Identitätsbewusstsein mithilfe von Conscious-Rap-Musik zu fördern versucht. Dieses Projekt wird nicht im Kontext eines bestimmten Unterrichtsfaches durchgeführt, sondern als extracurriculares Projekt. Für die Kommunikation in der Arbeitsgemeinschaft ist folgende sprachliche Regelung vorgesehen: Untereinander können die Schüler*innen in jeglicher Sprachform sprechen, die für die Bewältigung der Aufgabe nützlich ist. Als einzige strenge Regel gilt $\mathrm{zu}$ beachten, dass niemand in der Gruppe bewusst durch Sprache ausgeschlossen oder diskriminiert werden darf. Für Kommunikation in der gesamten Gruppe bedeutet dies, dass in der Sprache gesprochen wird, in der sich alle zufriedenstellend ausdrücken können. Die Arbeitsanweisungen auf Arbeitsblättern sind daher im Folgenden auf Englisch formuliert, da das Projekt im Kontext einer Londoner Schule entstand.

Folgende Ankündigung erhalten die Schüler*innen vor Beginn des Projekts. Sie stellt kurz und knapp Ziel und Kontext dar und lädt die Schüler*innen dazu ein, teilzunehmen.

On the $19^{\text {th }}$ of December, our school celebrates the Day of Cultures and Languages. All pupils are invited to participate by making an artistic contribution, which shows their multicultural and multilingual identities. Performances can take the form of spoken word poetry (poems recited aloud) or rap songs. Pupils should perform in groups of 2-4. There are three important rules for your performance:

1) No insults, no bullying!

2) Stay true to yourselves. Nothing is worse than being fake!

3) Put across a message about yourselves and your communities!

We are looking forward to your performances!

Abbildung 2: Ankündigung des Projekts

Im Mittelpunkt des Projekts steht die Schaffung eines künstlerischen Beitrags, der die multikulturellen und multilingualen Identitäten der Schüler*innen 
repräsentiert.

Die Ankündigung hebt drei wichtige Regeln hervor, die kurz erläutert werden sollen: (1) Beleidigungen und Mobbing sind nicht zulässig, da die Schüler*innen sich in einem sicheren Raum fühlen sollen, in dem sie sich frei und ohne Angst vor Diskriminierung äußern können. Dies hat mit Regel (2) zu tun, die von ihnen verlangt, sich selbst treu zu bleiben und ihre echte Persönlichkeit in die Aufgabe einzubringen. Soll die Lernaufgabe emanzipatorische Identity Awareness fördern, so ist es notwendig, dass die Schüler*innen ihre eigenen Erfahrungen und Einstellungen einbringen, also authentisch sind. Zudem ist diese Regel eine viel besungene Regel der HipHop-Gemeinschaft. Regel (3) schafft die Verbindung zwischen Individualität und Sozialität: Die Schüler*innen werden dazu aufgefordert, sich im Kontext ihrer Umwelt zu identifizieren, so wie es Rapper*innen im Conscious Rap tun.

Das Projekt nimmt den folgenden Verlauf:

\begin{tabular}{|c|c|c|c|}
\hline & Phase & Performative elements & Material \\
\hline 1 & $\begin{array}{l}\text { Getting to know each } \\
\text { other }\end{array}$ & $\begin{array}{l}\text { P1: ball of wool } \\
\text { P2: vowels }\end{array}$ & \\
\hline 2 & Exploring one's identity & P3: my language sculpture & $\begin{array}{l}\text { M1: self-observation } \\
\text { M2: language portrait }\end{array}$ \\
\hline 3 & $\begin{array}{l}\text { Exploring languages and } \\
\text { power }\end{array}$ & $\begin{array}{l}\text { P4: small power play } \\
\text { P5: the Welsh Not } \\
\text { P6: the power of insults } \\
\text { P7: inappropriate greetings }\end{array}$ & M3: power and language \\
\hline 4 & $\begin{array}{l}\text { Exploring one's } \\
\text { community }\end{array}$ & $\begin{array}{l}\text { P8: reenactment } \\
\text { P9: community collage }\end{array}$ & M4: observation \\
\hline 5 & $\begin{array}{l}\text { Exploring one's position } \\
\text { in one's community }\end{array}$ & $\begin{array}{l}\text { P10: my language sculpture } \\
\text { (again) }\end{array}$ & M5: self-reflection \\
\hline 6 & $\begin{array}{l}\text { Understanding street } \\
\text { poetry and rhyme }\end{array}$ & $\begin{array}{l}\text { P11: press conference } \\
\text { P12: language sculpture }\end{array}$ & $\begin{array}{l}\text { M6: analysis of multilingual } \\
\text { rap }\end{array}$ \\
\hline 7 & $\begin{array}{l}\text { Developing a collective } \\
\text { performance }\end{array}$ & $\begin{array}{l}\text { P13: beats and rhymes } \\
\text { P14: rehearsals }\end{array}$ & $\begin{array}{l}\text { M7: creating a rap / } \\
\text { spoken word } \\
\text { performance }\end{array}$ \\
\hline 8 & Final Performance & & M8: performing on \\
\hline
\end{tabular}

Abbildung 3: Verlauf des Projekts

Es gliedert sich damit in acht Phasen, die im Folgenden grob den Dimensionen der Kompetenz Sprachenbewusstheit zugeordnet werden sollen:

Das Material für die Phasen 6 und 7 des Projekts befindet sich ausschnittweise in Anhang A und B. Bei Interesse an weiterem Material kann der Autor dieses Artikels per E-Mail kontaktiert werden.

Bei der Umsetzung in die Praxis an einer Londoner Schule hat sich zudem der folgende Aspekt als wichtig herausgestellt: Für die 7. Phase, in der die Schüler*innen ihre eigenen Performances kreieren, ist es lohnenswert, nicht nur die sprachliche und rhythmische, sondern auch die körperlich-räumliche Gestaltung zu planen. Es gibt den Schüler*innen Selbstbewusstsein für die Performance, wenn sie sich darauf vorbereiten, wie sie den Raum nutzen und 
Tabelle 2: Darstellung des Projekts in Bezug auf die Förderung von Sprachenbewusstheit

\begin{tabular}{|c|c|c|c|c|}
\hline & Dimensionen & Perspektiven & Sprachebenen & Wissensebenen \\
\hline \multirow[t]{2}{*}{1} & affektiv, soziokulturell & Ich + Gesellschaft & interlingual & Einstellungen \\
\hline & \multicolumn{4}{|c|}{$\begin{array}{l}\text { Die Schüler*innen lernen sich gegenseitig in zwei performativen Spielen (P1 und P2) kennen, in die sie ihr } \\
\text { Sprachrepertoire einfließen lassen. Dies dient der Schaffung eines Gemeinschaftsgefühls als sprachlich } \\
\text { heterogene Gruppe und von Neugierde auf die sprachliche Identität der anderen. }\end{array}$} \\
\hline \multirow[t]{2}{*}{2} & $\begin{array}{l}\text { sprachstrukturell, } \\
\text { affektiv, soziokulturell }\end{array}$ & Ich & interlingual & $\begin{array}{l}\text { Einstellungen, Fähigkeit } \\
\text { (Perspektivübernahme) }\end{array}$ \\
\hline & \multicolumn{4}{|c|}{$\begin{array}{l}\text { In der zweiten Phase erkunden die Schüler*innen ihre individuelle sprachliche Identität (M1). Dafür fertigen sie } \\
\text { unter anderem ein individuelles Sprachenporträt (M2) an und drücken ihre Identität mithilfe von } \\
\text { Mitschüler*innen in Form einer Menschenskulptur (P3) aus. }\end{array}$} \\
\hline \multirow[t]{2}{*}{3} & $\begin{array}{l}\text { sprachstrukturell, } \\
\text { affektiv, soziokulturell, } \\
\text { politisch }\end{array}$ & Gesellschaft + Ich & $\begin{array}{l}\text { intralingual, interlingual, } \\
\text { metalingual }\end{array}$ & $\begin{array}{l}\text { Wissen, Einstellungen, } \\
\text { Fähigkeiten }\end{array}$ \\
\hline & \multicolumn{4}{|c|}{$\begin{array}{l}\text { In der dritten Phase werden Fragen von Sprache und Macht in den Vordergrund gestellt. Im Material } 3 \text { werden } \\
\text { verschiedene Aspekte behandelt, wie z.B. die Definition von Macht allgemein, Gewalt durch Sprache (in } \\
\text { Beleidigungen) und die Hierarchien zwischen Sprachen als Mittel der Erlangung von beruflichem Erfolg oder } \\
\text { Anerkennung bei Freunden. Die performativen Übungen 4-7 beziehen die Erfahrungs- und Gefühlswelt der } \\
\text { Schüler*innen mit ein. Der Rapsong, "Esperanto" wird in Zusammenhang mit einem Zeitungsartikel über die } \\
\text { Plansprache behandelt. }\end{array}$} \\
\hline \multirow[t]{2}{*}{4} & soziokulturell, politisch & Gesellschaft & intralingual, interlingual & Fähigkeit (Analyse) \\
\hline & \multicolumn{4}{|c|}{$\begin{array}{l}\text { Phase } 4 \text { leitet den Blick auf die Umwelten der Schüler*innen, indem sie in M4 Beobachtungsaufträge erhalten. } \\
\text { Die performativen Übungen 8-9 dienen dazu, die Beobachtungen im Klassenverband erfahrbar zu machen, zu } \\
\text { sichern und zu vertiefen. }\end{array}$} \\
\hline \multirow[t]{2}{*}{5} & soziokultt & Ich + Gesellschaft & interlingual & \\
\hline & \multicolumn{4}{|c|}{$\begin{array}{l}\text { Phase } 5 \text { stellt die Verbindung zwischen Individualität und Sozialität her. Die Erkenntnisse aus den Phasen 2-4 } \\
\text { werden durch gezielte Fragestellungen in M5 aus individueller Perspektive reflektiert. Ihren Erkenntnisprozess } \\
\text { geben die Schüler* in der Wiederholung der Sprachenskulptur (P3 aus Phase 2) (P10) wieder. Zu diesem } \\
\text { Zeitpunkt ist zu erwarten, dass das Bewusstsein und die Einstellung gegenüber der eigenen Identität und } \\
\text { fremder Identitäten vor dem Hintergrund soziokultureller und politischer Fragen gewachsen und affektiv } \\
\text { gestärkt worden sind. }\end{array}$} \\
\hline \multirow[t]{2}{*}{6} & alle I & Gesellschaft & $\begin{array}{l}\text { erlingual, translingual, } \\
\text { etalingual }\end{array}$ & $\begin{array}{l}\text { Wissen, Fähigkeiten } \\
\text { (Analyse, } \\
\text { Perspektivübernahme) }\end{array}$ \\
\hline & \multicolumn{4}{|c|}{$\begin{array}{l}\text { In Phase } 6 \text { analysieren die Schüler*innen in Gruppenarbeit Rapsongs (darunter,Viêt Kiều“). Im Mittelpunkt } \\
\text { steht die Erarbeitung der makro- und mikrosprachlichen Struktur der Songs sowie die Charakterisierung der in } \\
\text { den Raps repräsentierten Identitäten (M6). Dabei greifen die Schüler* auf alle Dimensionen der } \\
\text { Sprachenbewusstheit zurück, um die Intersektion der Identität zufriedenstellend zu untersuchen. Mithilfe der } \\
\text { performativen Übungen } 11 \text { und } 12 \text { teilen sie die gewonnenen Erkenntnisse im Klassenverband und heben sie in } \\
\text { der gemeinsamen Reflexion auf eine Metaebene. }\end{array}$} \\
\hline \multirow[t]{2}{*}{7} & $\begin{array}{l}\text { alle Dimensionen (inkl. } \\
\text { poetisch) }\end{array}$ & Ich + Gesellschaft & $\begin{array}{l}\begin{array}{l}\text { intralingual, interlingual, } \\
\text { translingual }\end{array} \\
\end{array}$ & $\begin{array}{l}\text { Einstellungen, Fähigkeit } \\
\text { (Produktion) }\end{array}$ \\
\hline & \multicolumn{4}{|c|}{$\begin{array}{l}\text { In Phase } 7 \text { werden die Schüler*innen schließlich selbst aktive Produzenten von identitätsbewusster } \\
\text { Sprachkunst. In mehreren Schritten (M7), die ihnen Inhalte aus den vergangenen Phasen in Erinnerung rufen } \\
\text { sowie Möglichkeiten des Reimens und der Strukturierung ihrer Performance aufzeigen, kreieren sie eine } \\
\text { Mischung aus individueller und kollektiver Performance in ihren Gruppen. }\end{array}$} \\
\hline & affektiv & Ich + Gesellschaft & interlingual, translingual & Einstellungen \\
\hline & \multicolumn{4}{|c|}{$\begin{array}{l}\text { Die finale Performance bildet den abschließenden Höhepunkt des Projekts: die mehrsprachigen und } \\
\text { mehrkulturellen Identitäten der Schüler*innen werden präsentiert. Viele offene Fragen darüber, wie die } \\
\text { Schüler*innen sich in und außerhalb der Schule im Bewusstsein ihrer vollständigen, hybriden Identitäten in } \\
\text { Zukunft verhalten, werden anschließend individuell und im Klassenverband reflektiert (M8). Dabei wird klar, } \\
\text { dass die poetische Performance in der Lernaufgabe nur der Anfang eines stetigen Prozesses bewussten, kritisch- } \\
\text { kreativen sprachlichen Handelns in gesellschaftlichen Kontexten sein kann. }\end{array}$} \\
\hline
\end{tabular}


wie sie sich zueinander positionieren werden: Wie stehen sie zum Publikum? Bewegen sie sich? In einer bestimmten Formation? Im Laufe des Projektes erstellen die Schüler*innen Skulpturen, die ihre eigenen oder verschiedene sprachliche Identitäten repräsentieren (P3, P9, P10, P12). Sie sollten dazu angeregt werden, bewusst auszuwählen, welche Gestaltungsmittel sie daraus für ihre kollektive Performance übernehmen wollen.

Ein Ergebnis dieses Projekts, das kürzlich an einer Schule in London durchgeführt wurde, kann in Anhang $C$ nachgelesen werden. Vier 16-jährige Schüler arbeiteten darin an einer gemeinsamen Performance, die die Sprachen Englisch, Deutsch, Spanisch, Französisch, Arabisch, Chinesisch und Elemente der so genannten Hip-Hop-Nation-Language enthält. Die Sprachen Arabisch, Chinesisch und Deutsch sind neben Englisch jeweils Teil des familiär erlernten Sprachrepertoires von drei Schülern. Der vierte Schüler ist in einsprachiger familiärer Umgebung (englisch) aufgewachsen. Alle Schüler nehmen des Weiteren am schulischen Deutsch- und Französischunterricht teil, zwei von ihnen außerdem jeweils an Chinesisch- oder Spanisch-Unterricht.

Das Produkt zeigt, dass die Schüler alle Sprachen eng miteinander verbunden haben. Die Sprachen Englisch, Deutsch und Französisch dominieren und werden bunt vermischt, während Chinesisch, Spanisch und Arabisch weniger Platz in der Performance einnehmen. Die Schüler reflektierten diese Entscheidung mit der Begründung, dass sie einen gemeinsamen Rap erstellen wollten, den sie untereinander problemlos verstehen und auch ihren Mitschülern leicht verständlich machen konnten. Sie entschieden sich dagegen, individuelle Verses zu verfassen.

In dieser Hinsicht gibt dieses Produkt Aufschluss darüber, dass insbesondere das kollektive Selbstbewusstsein Stärkung erfahren hat. Dies liest man auch aus den Lyrics heraus (siehe Anhang C) und spiegelte sich auch in der Performance wider: Den Hook rappten alle gleichzeitig zusammen, während die Schüler sich die Lines in den beiden Verses untereinander aufteilten. Das letzte Wort von jedem Verse (,zusammen“ und „Go!") wurde gemeinsam gerappt. Dabei stellten sich die Schüler in einem Halbkreis auf, den ein Schüler mit einem Schritt nach vorn dann betrat, wenn er individuell rappte. Beim Rappen imitierten sie für den Rap typische Handbewegungen, während die gerade nicht Rappenden den Kopf zum Beat wippen ließen. Die Gesamtperformance aus sprachlicher Mischung und Choreographie erweckte den Eindruck, dass die individuellen Schüleridentitäten zwar wichtig für die Performance waren, aber in den Hintergrund des Gemeinsamen und Verbindenden traten, das etwas Neues, Harmonisches und Hybrides bildete.

\section{Resümee}

Rap ist eine identitätsstiftende Praxis, die sich als solche bewusst ist. Die Rapsongs von Fawng Daw \& Co sind absichtsvolle Kreationen, mehrfach überarbeitet, durchdacht und erprobt. Sie sind Performances, entspringen aber der Lebenswelt der Rapper*innen und machen daher deutlich: Auch im Alltag 
können wir bewusst entscheiden, wie wir uns verhalten. Alles, was wir tun, wie wir uns kleiden, wo und was wir essen, mit wem wir uns treffen, wie wir uns vergnügen, welche Sprachen wir auf welche Art sprechen etc., all dies sind letztendlich Performances unserer Identitäten.

Das Potenzial von Performativität für den Sprachenunterricht ist damit zweierlei: Erstens macht es schlichtweg Spaß, holistisch zu lernen. Indem Schüler*innen performative Übungen durchführen und selbst zu Performer*innen werden, nutzen sie Körper, Gehirn und Seele zugleich, sodass Lernen zu einem Erlebnis wird, das nicht selten von Flow-Gefühlen begleitet wird. Diese Art von Lernen verankert sich tiefer im Gedächtnis.

Zweitens bedeutet Performance die kognitiv bewusste, aber gleichzeitig affektive Auseinandersetzung mit der eigenen Identität. Performativität bildet hierbei die Brücke zwischen kognitivem Bewusstsein und affektivem Zugang. Auf affektiver Ebene erlernen die Schüler*innen positive Akzeptanz ihrer eigenen sprachlichen Fähigkeiten und Eigenschaften und der ihrer Mitschüler*innen. Sie erhalten Gelegenheit, Stolz für sich selbst und ihre vielfältige Lerngruppe zu empfinden. Die soziale Bezugsnorm, die sich oft in Form von Konkurrenzdruck äußert, weicht einem wachsenden individuellen und kollektiven Selbstwertgefühl. Auf kognitiver Ebene wird das Wachstum dieses Selbstwertgefühls vom Wachstum eines Selbst- und Fremdbewusstseins begleitet. Die Angst vor dem Fremden und Anderen sowie die Scham vor dem eigenen Ungenügendsein werden abgebaut, indem die Lernenden erfahren, dass Definitionen von Anderssein und Gutsein bestimmten sozialen Konventionen und Hierarchien unterliegen, die nicht naturgegeben sind.

Diese Einblicke würden ihnen genommen, wenn sie von der Institution Schule verordnet bekämen, wie sie sich angemessen $\mathrm{zu}$ verhalten und $\mathrm{zu}$ sprechen haben. Eine Schule, die soziale Regeln und Hierarchien unhinterfragt reproduziert, löst bei vielen Schüler*innen das Gefühl aus, bevormundet zu werden und aufgrund ihrer von Standardnormen abweichenden Identitäten minderwertig zu sein. Sprache, Ethnie, Gender und Schicht sind miteinander verwoben. Gibt man Schüler*innen vor, ihr sprachliches Handeln entsprechend hegemonialer Maßstäbe ändern zu müssen, so kann dies für sie eine Bedrohung der Schnittmenge ihrer gesamten Identität bedeuten.

Im Gegensatz dazu birgt die Schaffung von Identity Texts, in denen Schüler*innen ihre Identitäten ausdrücken können und durch Interaktion mit dem Publikum oder Mitschüler*innen Bestätigung erhalten, ein enormes transformatorisches Potenzial. In dem in London durchgeführten Projekt führte dies vor allem zu einem stärkeren Zusammengehörigkeitsgefühl zwischen den Mitschülern.

Die Bestätigung der diversen Identitäten der Schüler*innen in einer Gruppe ist nach Ansicht dieses Autors der erste Schritt in Richtung eines positiven Selbstbewusstseins, das es ihnen erlaubt, auch kritisch ihre eigenen Identitäten $\mathrm{zu}$ betrachten und darüber nachzudenken, inwiefern ihr eigener Habitus im Sinne von Bourdieu sozial konstruiert ist und was sie gern selbst lernen möchten, um ihre Identitäten zu verändern, vertiefen und erweitern. Die 
Selbstinszenierung im gesprochenen Wort schärft ihr Bewusstsein dafür, dass sie ihre Identitäten kontrollieren können. Anstatt Produkte externer Zuschreibungen, Diskurse und Erziehungsmaßnahmen zu sein, werden sie damit zu Urhebern ihrer eigenen Identität, zu Subjekten.

\section{Bibliografie}

Alim, H. Samy (2009b): Intro. In: Alim, H. Samy; Ibrahim, Awad \& Pennycook, Alastair (Hrsg.): Global linguistic flows. Hip Hop cultures, youth identities, and the politics of language. New York: Routledge, 1-22

Bausch, Karl-Richard (2003): Zwei- und Mehrsprachigkeit. Überblick. In: Bausch, Karl-Richard (Hrsg.): Handbuch Fremdsprachenunterricht. 4. Aufl. Tübingen: Francke, 439-445

Blutner, Stefan (im Erscheinen): Mit mehrsprachigem Rap kritische Sprachenbewusstheit fördern. Master-Arbeit 2017. Freie Universität Berlin. Dokumenten-Server

Breidbach, Stephan; Elsner, Daniela \& Young, Andrea (2011): Language awareness in teacher education. Cultural-political and social-educational dimensions. In: Breidbach, Stephan; Elsner, Daniela \& Young, Andrea (Hrsg.): Language awareness in teacher education. Cultural-political and social-educational perspectives. Frankfurt am Main: Peter Lang (Mehrsprachigkeit in Schule und Unterricht), 11-19

Dalzell, Tom (2014): Hip-Hop Slang. In: Coleman, Julie (Hrsg.): Global English Slang. Methodologies and Perspectives. London: Routledge, 15-24 Daw, Fawng (2012): Vịt Ki`u. https: //youtu.be/W_V2JFKr50 [letzter Zugriff 8. Februar 2018]

Ellis, Elizabeth M. (2012): Language awareness and its relevance for TESOL. In: University of Sydney Papers in TESOL 7, 1-23

García, Ofelia (2008): Multilingual language awareness and teacher education. In: Hornberger, Nancy H. (Hrsg.): Encyclopedia of language and education. 2. Aufl. Band 6. New York: Springer, 385-400

García, Ofelia \& Wei, Li (2014): Translanguaging. Language, bilingualism and education. Basingstoke: Palgrave Macmillan

Gogolin, Ingrid (2004): Lebensweltliche Mehrsprachigkeit. In: Bausch, Karl-Richard \& Königs, Frank G. (Hrsg.): Mehrsprachigkeit im Fokus. Arbeitspapiere der 24. Frühjahrskonferenz zur Erforschung des Fremdsprachenunterrichts. Tübingen: Narr (Giessener Beiträge zur Fremdsprachendidaktik), 55-61

Gogolin, Ingrid (2010): Chancen und Risiken nach PISA. Über Bildungsbeteiligung von Migrantenkindern und Reformvorschläge. In: Auernheimer, Georg (Hrsg.): Schieflagen im Bildungssystem. Wiesbaden: Springer Fachmedien (Interkulturelle Studien), 33-50 
Hawkins, Eric (1984): Awareness of Language. An Introduction. Cambridge: Cambridge University Press

Hu, Adelheid (2003): Schulischer Fremdsprachenunterricht und migrationsbedingte Mehrsprachigkeit. Tübingen: Narr (Giessener Beiträge zur Fremdsprachendidaktik)

Lensch, Juliane (2014b): Zur musikalischen Charakteristik eines Rap. In: Prinz, Manfred (Hrsg.): RapRoMania. Jugendkulturen und Fremdsprachenunterricht. Spanisch/Französisch. Stuttgart: ibidem (Romanische Sprachen und ihre Didaktik, 48), 49-50

Low, Bronwen \& Sarkar, Mela (2014): Translanguaging in the Multilingual Montreal Hip-Hop Community. Everyday Poetics as Counter to the Myths of the Monolingual Classroom. In: Blackledge, Adrian \& Creese, Angela (Hrsg.): Heteroglossia as practice and pedagogy. Dordrecht: Springer (Educational Linguistics, 20), 99-118

Lüdtke, Solveig (2007): Globalisierung und Lokalisierung von Rapmusik am Beispiel amerikanischer und deutscher Raptexte. Berlin: LIT-Verl. (Musik und Text, 2)

Oomen-Welke, Ingelore \& Dirim, Inci (2013): Mehrsprachigkeit in der Klasse wahrnehmen - aufgreifen - fördern. Einleitung zu diesem Band. In: Oomen-Welke, Ingelore \& Dirim, Inci (Hrsg.): Mehrsprachigkeit in der Klasse wahrnehmen - aufgreifen - fördern. Stuttgart: Klett, 7-24

Pennycook, Alastair (2003): Global Englishes, Rip Slyme, and performativity. In: Journal of Sociolinguistics 7/4, 513-533

Tracy, Rosemarie (2014): Mehrsprachigkeit. Vom Störfall zum Glücksfall. In: Krifka, Manfred; Błaszczak, Joanna; Leßmöllmann, Annette; Meinunger, André; Stiebels, Barbara; Tracy, Rosemarie \& Truckenbrodt, Hubert (Hrsg.): Das mehrsprachige Klassenzimmer. Berlin, Heidelberg: Springer, 12-33

Wolff, Dieter (2010): Spracherwerb und Sprachbewusstheit. Sind mehrsprachige Menschen bessere Sprachenlerner? In: Cuadernos de Filología Alemana 2, 177-190 


\section{A Anhänge}

\section{A.1 Anhang A: Arbeitsblätter zur Phase 6 des Projekts (Ausschnitte)}

Aufgrund von Platzgründen können hier nicht alle Arbeitsblätter zur Verfügung gestellt werden. Bei Interesse wenden Sie sich gern per E-Mail an den Autor dieses Artikels.

\section{A.2 Anhang B: Arbeitsblätter zur Phase 7 des Projekts}

\section{A.3 Anhang C: Ein Ergebnis des Projekts in London}




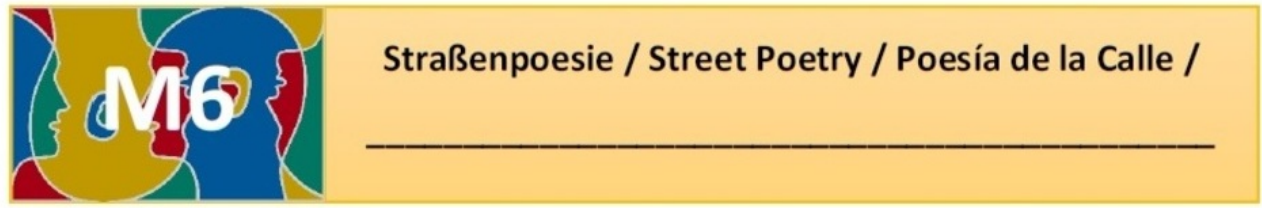

\section{Task 4 Listen to the rap song "Việt Kiều" and answer the following questions:}

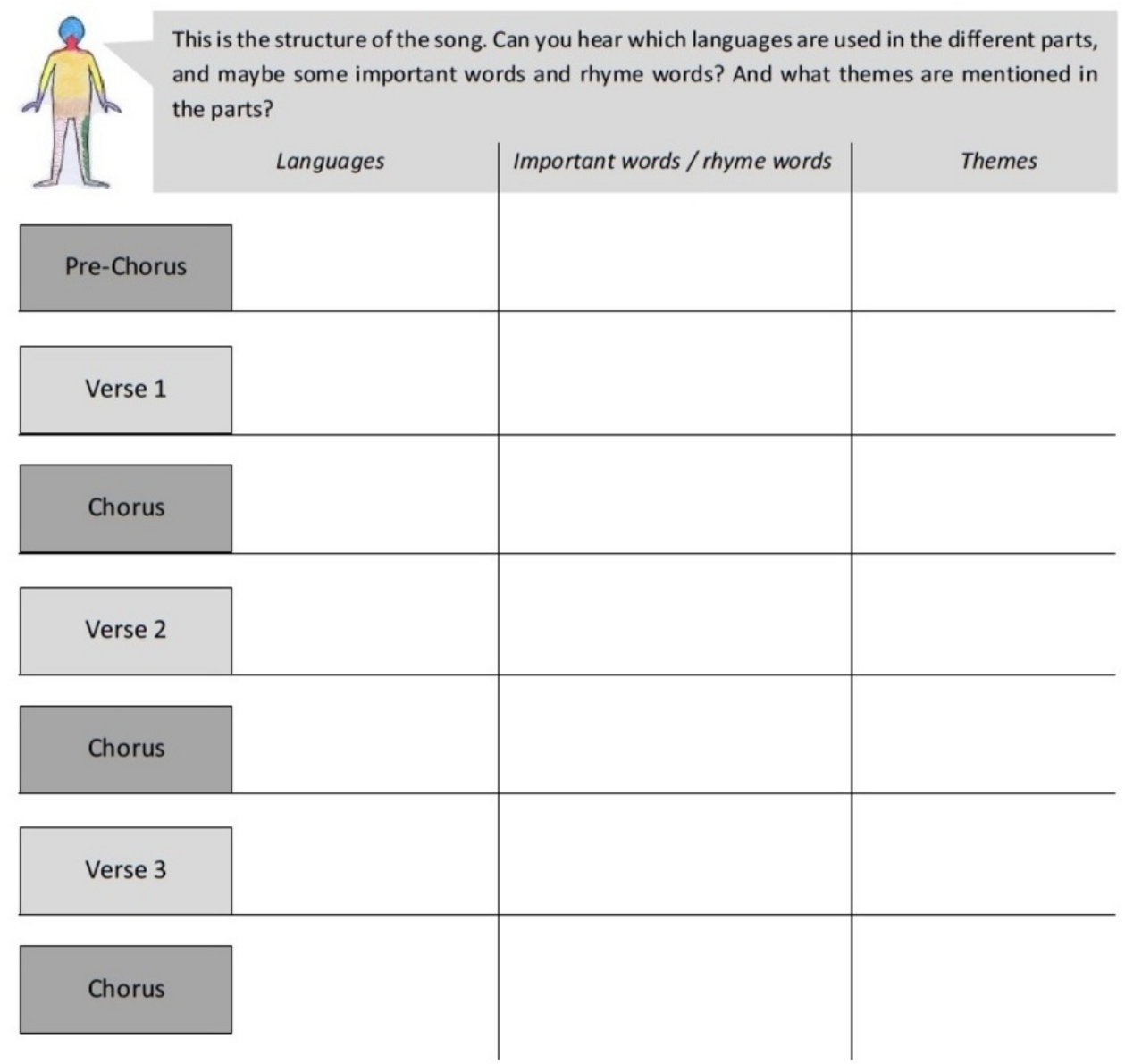

Task 5 Take a look at the chorus.

- Which variety of English is used? Give examples.

- What kind of rhyme scheme do we find?

- What might "Việt Kiều" mean?

- Why does he sing "Stop the hate"?
Born in the east, raised in the west,

heart of the motherland pounding in my chest. I'm a Việt Kiều.

Yeah, I'm a Việt Kiều. Kiều, Kiều, Kiều.

Stop the hate, let me do my thang.

A child of two worlds: I am who I am.

There is nothing I need to change cuz I am a...

Việt Kiều.

Abbildung 4: 


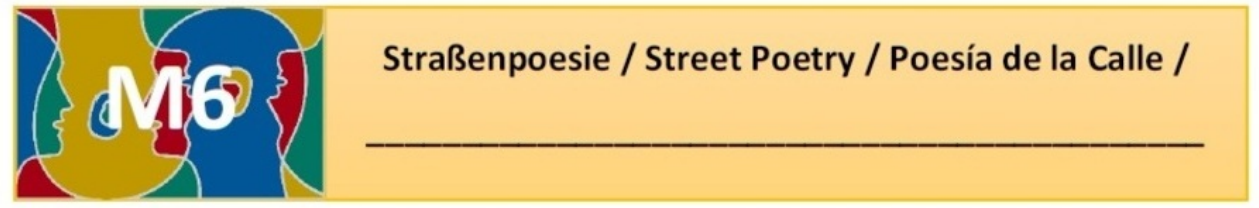

Task 5

Take a look at the whole song and sum up the content of the verses on the right margin of the paper. Use symbols and bullet points.

\title{
Fawng Daw - "Việt Kiều"
}

Pre-Chorus
Born in the east, raised in the west,

heart of the motherland pounding in my chest.

I'm a Viet Kieu.

Yeah, I'm a Viet Kieu, Kieu, Kieu, Kieu.

Verse 1

5 Vietnamese immigrant, Germany's citizen,

moved to the USA, currently resident.

Proud of my heritage, coming to represent.

Gotta get Benjamins for all of my relatives.

Shed blood, sweat and tears, making my nation proud.

10 Better put ya hands up high, this is for the Asian crowd.

Gotta get famous now, ain't nothin's stopping me.

You hear the stomach growl? Homie, we gotta EAT.

I'm just a skinny boy, a Hanoi City boy.

Grindin' in Caliornia, even though I'm unemployed.

15 Catch me at movie sets, studios, or photo shoots.

Try to provide cuz thats what a man supposed to do.

Brother, tell me do you know how this feels?

They keep on staring at me, what's the big deal?

I'm just a westernized Easterner with skillz.

20 I am someone you call a Viet Kieu.

Chorus

Born in the east, raised in the west,

heart of the motherland pounding in my chest.

I'm a Viet Kieu.

Yeah, I'm a Viet Kieu, Kieu, Kieu, Kieu.

25 Stop the hate, let me do my thang.

A child of two worlds: I am who I am.

There is nothing I need to change cuz I am a...

Verse 2

Viet Kieu, nhung ma huong cua toi gian toi ve que huong

Cho nen ten toi moi co nghia la "Viet Phuong"

30 Nhung y cua toi muon noi khong co phai la toi dac biet

Toi chi hoi khac biet, cach suy nghi khac viet

Toi la mot nguoi chau a song ochau lau dai

Va toi di nuoc nao cung bi ho goi la nguoi nuoc ngoai

Yeah homie, toi la toi la nguoi Viet Kieu

35 Bo me lam viec nhieu, cho nen toi biet dieu

Biet dieu, gotta buy my mom and dad a big crib,

overlooking the ocean on top of a big hill,

Benjamins $=100$ Dollar notes (Benjamin Franklin)

because they gave their life to give me paradise.

I couldn't pay the price to match their sacrifice.

40 They gave me all the tools, so when I get the millions

I'll go back to their village and give it to all the children.

Cuz I'm a Viet Kieu, a child of 2 worlds,

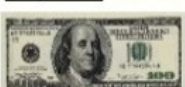

Using my given talents to serve.

\author{
Viet Kieu, but I want to go back to my home country, \\ so my name means "Direction Vietnam". \\ But I don't think I'm special, \\ Pm just a little different \\ because I lived in Europe for a long time, \\ which means I am a foreigner wherever I go. \\ Yeah homie, I am a Viet Kieu \\ my parents work a lot, so I know how to behave. \\ Behave, gotta buy my mom and dad a big crib.
}

Abbildung 5: 


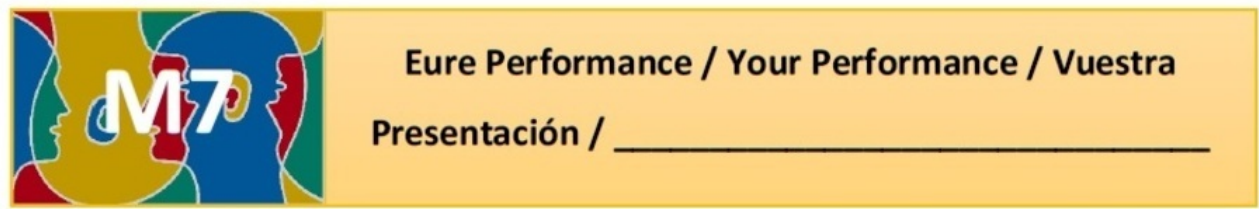

Find your group identity. What do you have in common? What makes you a group? What makes

Task 1 you strong together? What makes you special? What goals and values do you share? What kind of message would you like to transmit to the world? Write down key words and ideas.

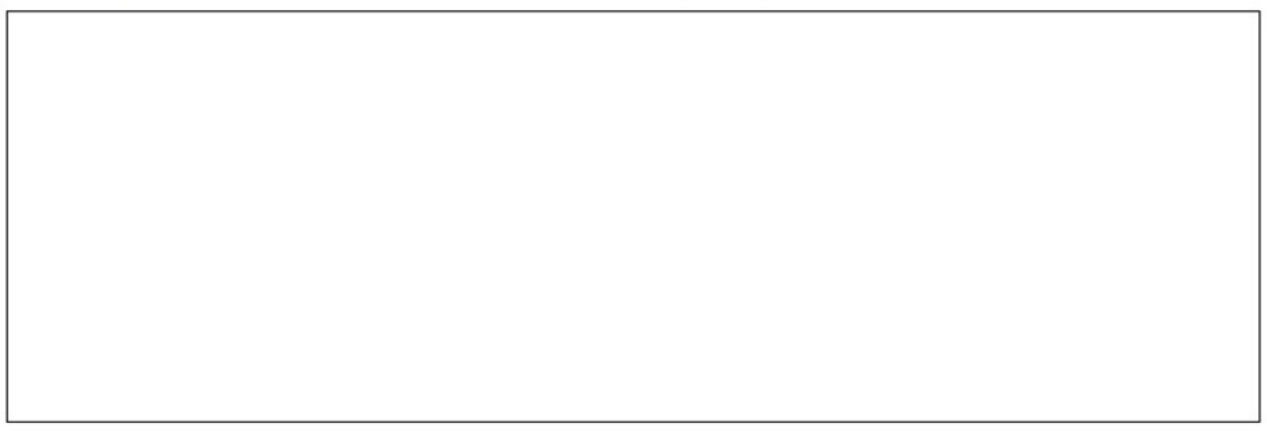

Task 2 Choose 2-3 keywords and Find as many rhyme words or rhyming word groups in the languages spoken by all of you.
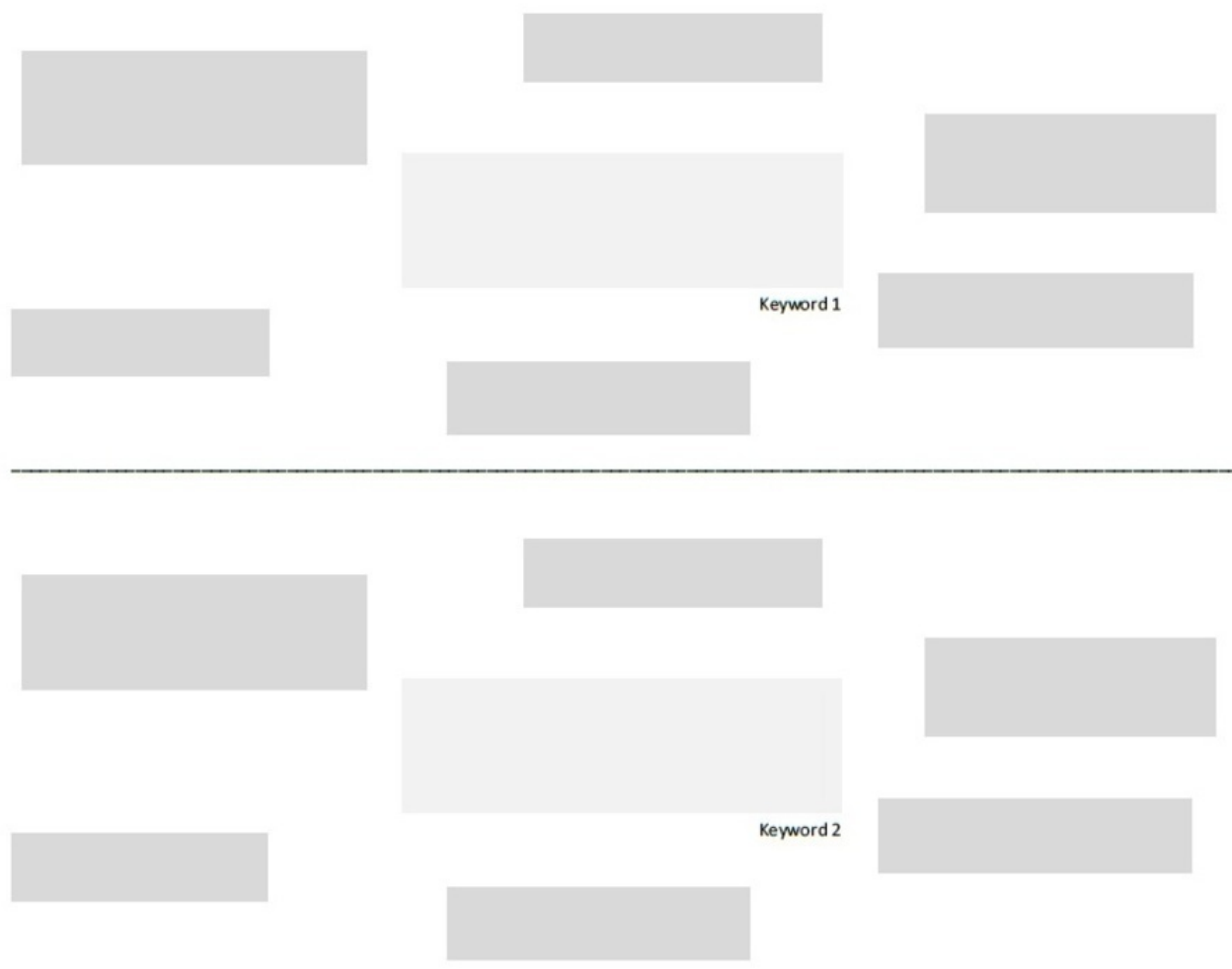
Task 3 Based on tasks 1 and 2, write a chorus for your rap song / spoken word performance.

\begin{tabular}{|l|l|}
\hline 1 & \\
\hline 2 & \\
\hline 3 & \\
\hline 4 & \\
\hline 5 & \\
\hline 6 & \\
\hline 7 & \\
\hline 8 & \\
\hline$-\cdots$ & \\
\hline
\end{tabular}

Task 4 If you want to create a rap song, look online (e.g. youtube) for a beat that fits your chorus. Everybody will have to follow this beat in their individual verses, too.

Task 5 Make a scheme for your song/performance.

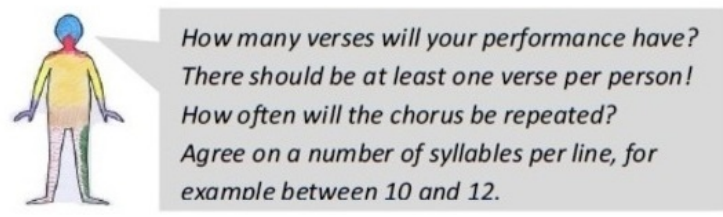




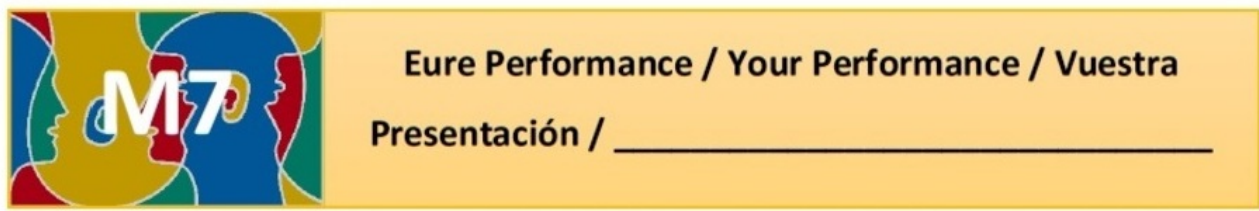

Task 6

Collect ideas for your individual verse. Take a look back at materials 1-6. And think about the following questions. You do not have to answer them, but they can inspire you.

\begin{tabular}{|c|c|}
\hline $\begin{array}{l}\text { What part of your } \\
\text { (linguistic) identity are you } \\
\text { proud of? }\end{array}$ & \\
\hline $\begin{array}{l}\text { What feelings do you have } \\
\text { about your languages? }\end{array}$ & \\
\hline $\begin{array}{l}\text { How are your languages } \\
\text { related to each other? }\end{array}$ & \\
\hline $\begin{array}{l}\text { Which struggles are you } \\
\text { facing? }\end{array}$ & \\
\hline $\begin{array}{l}\text { What are your values, } \\
\text { attitudes and goals? }\end{array}$ & \\
\hline $\begin{array}{l}\text { What do you want to } \\
\text { change about yourself? }\end{array}$ & \\
\hline $\begin{array}{l}\text { What do you want to } \\
\text { change about the world? }\end{array}$ & \\
\hline Who can you count own? & \\
\hline $\begin{array}{l}\text { Who or what are you } \\
\text { resisting against? }\end{array}$ & \\
\hline $\begin{array}{l}\text { What would be a good } \\
\text { term to describe your } \\
\text { identity in a few } \\
\text { (multilingual) words? }\end{array}$ & \\
\hline
\end{tabular}


Task 7 Write down keywords for your verse and find rhyming words.

\begin{tabular}{|l|l|}
\hline & \\
\hline & \\
\hline & \\
\hline & \\
\hline
\end{tabular}

Task 8 Compose your verse.

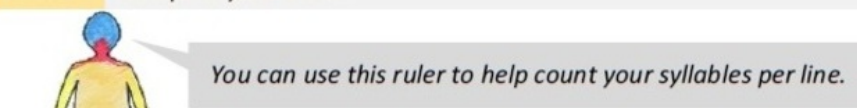

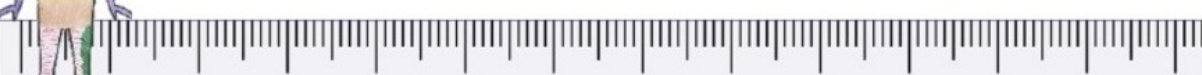

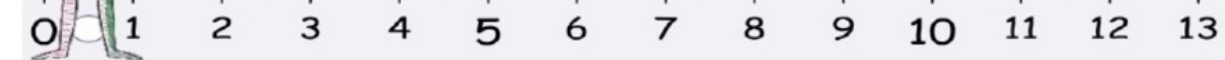

\begin{tabular}{|l|l|}
\hline 1 & \\
\hline 2 & \\
\hline 3 & \\
\hline 4 & \\
\hline 5 & \\
\hline 6 & \\
\hline 7 & \\
\hline 8 & \\
\hline 9 & \\
\hline 10 & \\
\hline 11 & \\
\hline 12 & \\
\hline$\cdots$ & \\
\hline
\end{tabular}




\section{Verse I}

Yo, listen up, Mann!

Welcome to our language club, Mann!

Toutes les langues sind hier willkommen. Unsre Vielfalt macht uns strong!

\section{We are many, but we're one!}

Also hört jetzt endlich zu, Mann

Yalla, Bruder, on chante, zusammen!

\section{$\operatorname{Hook}(x 2)$}

Ma langue, ta langue, notre langue! Unser Mix ist unser Flow, das ist unser Song! Wo bu zhidao what you're sayin',

But it's klar: We're stayin'

Piaoliang

is what makes us one!

\section{Verse II}

Todos somos diferentes personalidades.

Wir respektieren jeden, der in dieser Stadt ist. Respeto, respect, c'est le même mot In jeder Sprache: So join in our flow! Go!

\section{$\operatorname{Hook}(x 2)$}

\section{Legende:}

Hip-Hop-Nation-Language / Standard-Spanisch / Standard-Englisch / Standard-Deutsch /

Jugendsprachliches Deutsch/Umgangssprachliches Arabisch / Standard-Mandarin /

Standard-Französisch

\author{
Yo, hört zu, Mann! \\ Willkommen zu unserem Sprachclub, Mann! \\ Alle Sprachen sind hier willkommen. \\ Unsre Vielfalt macht uns stark. \\ Wir sind viele, aber wir sind eins. \\ Also hört jetzt endlich zu, Mann! \\ Los jetzt, Bruder, wir singen zusammen!
}

Meine Sprache, deine Sprache, unsre Sprache. Unser Mix ist unser Flow, das ist unser Song! Ich weiß nicht, was du sagst, aber es ist klar: Wir bleiben! Schön ist, was uns zusammenhält.

Wir sind alle verschiedene Persönlichkeiten. Wir respektieren jeden, der in dieser Stadt ist. Respekt, Respekt, das ist das gleiche Wort In jeder Sprache: Also schließ dich unserem Flow an! Los geht's! 